\title{
Discrete rogue waves of the Ablowitz-Ladik and Hirota equations
}

\author{
Adrian Ankiewicz, ${ }^{1}$ Nail Akhmediev, ${ }^{1}$ and J. M. Soto-Crespo ${ }^{2}$ \\ ${ }^{1}$ Optical Sciences Group, Research School of Physics and Engineering, The Australian National University, Canberra, \\ Australian Capital Territory 0200, Australia \\ ${ }^{2}$ Instituto de Óptica, C.S.I.C., Serrano 121, 28006 Madrid, Spain
}

(Received 8 June 2010; published 11 August 2010)

\begin{abstract}
We show that the Ablowitz-Ladik equation, which is an integrable form of the discretized nonlinear Schrödinger equation, has rogue wave solutions in the form of the rational solutions. We show that there is a hierarchy of rational solutions and we derive the two lowest-order ones using the Hirota technique. More generally, we present rational solutions for the discrete Hirota equation which includes, as particular cases, both the discrete Ablowitz-Ladik equation and the discrete modified Korteweg-de Vries (mKdV) equation.
\end{abstract}

DOI: 10.1103/PhysRevE.82.026602

PACS number(s): 05.45.Yv, 42.65.Tg, 42.65.Wi

\section{INTRODUCTION}

The Ablowitz-Ladik (A-L) equation [1,2] is an integrable form of the discretized nonlinear Schrödinger equation (NLSE). As such, it has multiplicity of solutions which are analogous to solutions of the NLSE. Among the known ones, we can mention soliton and multisoliton solutions of the A-L equation. The close relation seems straightforward; but, in reality, there is much confusion over attempts to integrate the A-L equation. The difficulty is related to the nonlinear term, which is not the result of a direct discretization, but has some nonlocality involved. At this price, the equation becomes integrable.

Solutions of the A-L equation on a zero background level have been studied extensively. The inverse scattering technique, with zero boundary conditions for this equation, has been developed in Ref. [2]. In particular, the basic zero background one-soliton ("sech") and the two-soliton solutions have been given in [3]. The latter work also covers the case of the discrete Hirota equation. The inverse scattering technique for nonvanishing boundary conditions has been developed in [4]. $N$-dark soliton solutions of the A-L equation have been given in terms of the Casorati determinant in [5].

There have been many endeavors to use the inverse scattering technique [2], zero-curvature representation [6], and Darboux transformations $[7,8]$ for the A-L equation. Standard techniques allow us to obtain a certain class of solutions. Moreover, in the case of the A-L equation, they are not always entirely convenient, as pointed out in [9]. If we are interested in solutions that do not belong to a specific class, we can use nontraditional methods. In this regard, new solutions have been obtained by using the Hirota method $[3,10]$ or by guessing the form of the solutions from the NLSE case and substituting them directly into the A-L equation [11]. The standard form of the integrable A-L equation can be written as

$$
i \frac{\partial \psi_{n}}{\partial t}+\left(\psi_{n-1}+\psi_{n+1}\right)\left(1+\left|\psi_{n}\right|^{2}\right)-2 \psi_{n}=0,
$$

where $t$ is the continuous evolution variable (time or longitudinal spatial variable) and $n=0, \pm 1, \pm 2, \ldots$ are integers.

Our aim in this work is to analyze the existing solutions and derive other results for the A-L equation. In particular, we are interested in solutions that are located on a finite background. These could be either localized or periodic solutions. In the case of the NLSE, the latter are known to be related to modulation instability and to rogue waves [12]. In this work, we are interested in similar objects generated by the A-L equation. Generally, discrete breathers have a variety of applications [13]. In particular, discrete rogue waves can be used as spatial energy concentrators in arrays of nonlinear waveguides [14]. Thus, presenting these solutions may well find practical applications.

\section{INVARIANTS AND CONSERVED QUANTITIES}

To start with, let us list relevant invariants of the equation. For the A-L equation, a solution multiplied by a complex number of unit modulus produces another solution. This property is known as phase invariance. For localized solutions with zero background at infinity, i.e., when $n \rightarrow \pm \infty$, there is an "energy invariant" that is conserved. If the background is not a zero but has a finite value $q$, we can redefine energy since, with the usual definition, it would become infinite. It is also conserved. Namely, for a solution $\psi_{n}$, labeled by $j$ located on a background field $q$, we define

$$
Q^{(j)}=\sum_{n=-\infty}^{\infty} Q_{n}^{(j)}
$$

where

$$
Q_{n}^{(j)}=\frac{1}{2}\left(\psi_{n}^{(j)} \psi_{n+1}^{(j) *}+\psi_{n}^{(j) *} \psi_{n+1}^{(j)}\right)-q^{2} .
$$

Another conserved quantity is the momentum

$$
P^{(j)}=i \sum_{n=-\infty}^{\infty}\left(\psi_{n}^{(j)} \psi_{n+1}^{(j) *}-\psi_{n}^{(j) *} \psi_{n+1}^{(j)}\right) .
$$

The expression for the momentum is the same for solutions on zero or nonzero background.

Solutions of the A-L equation are translationally invariant relative to shifts along the $t$ axis. They are also translationally invariant relative to shifts along the $n$ axis by an integer number. It is less obvious that they are translationally invariant relative to real (noninteger) shifts along the $n$ axis. In- 
deed, all solutions presented below can be shifted by real number $n_{0}$. They then look different but are still solutions of the A-L equation. For this reason, we plot the solutions in a "continuous way," although the functions are defined only at the discrete sites.

\section{NARITA SOLUTION}

A rich family of solutions of the A-L equation on a background has been obtained by Narita in 1991 [10]. Although the initial description was "a soliton on a background," the family actually covers a wider variety of physical situations. A comprehensive analysis of it would be instructive for obtaining more complicated solutions. This family is given by

$$
\psi_{n}(t)=-q \frac{g(n, t)}{f(n, t)} \exp \left(2 i q^{2} t\right),
$$

where

$$
\begin{gathered}
g(n, t)=\cos \left[x_{1}(n, t)+i \theta_{1}\right]+G \cosh \left[x_{2}(n, t)+i \theta_{2}\right], \\
f(n, t)=\cos \left[x_{1}(n, t)\right]+G \cosh \left[x_{2}(n, t)\right] .
\end{gathered}
$$

The coefficient $G$ is found from

$$
G^{2}=-\frac{\sin \left(k_{1}\right) \sinh \left(\theta_{1}\right)}{\sin \left(\theta_{2}\right) \sinh \left(k_{2}\right)},
$$

and

$$
\begin{aligned}
& x_{1}=k_{1} n+w_{1} t+x_{10}, \\
& x_{2}=k_{2} n+w_{2} t+x_{20} .
\end{aligned}
$$

This family of solutions has five free parameters $q, k_{1}, k_{2}$, $x_{10}$, and $x_{20}$. The value $q$ sets the background amplitude. The two latter parameters are responsible for translations. For simplicity, we can set them to be zero $x_{10}=0, x_{20}=0$. Two other parameters, $w_{1}$ and $w_{2}$, depend on $q, k_{1}$, and $k_{2}$. If we define $y$ as

$$
y=\frac{2\left(q^{2}+1\right)}{\cosh \left(\theta_{1}\right)-\cos \left(\theta_{2}\right)},
$$

then

$$
\begin{aligned}
w_{1}= & y\left\{\sin \left(k_{1}\right) \sin \left(\theta_{2}\right) \sinh \left(k_{2}\right)+[1\right. \\
& \left.\left.-\cos \left(k_{1}\right) \cosh \left(k_{2}\right)\right] \sinh \left(\theta_{1}\right)\right\}, \\
w_{2}= & y\left\{\left[\cos \left(k_{1}\right) \cosh \left(k_{2}\right)-1\right] \sin \left(\theta_{2}\right)\right. \\
& \left.+\sin \left(k_{1}\right) \sinh \left(k_{2}\right) \sinh \left(\theta_{1}\right)\right\} .
\end{aligned}
$$

In order to find $\theta_{1}, \theta_{2}$, we need to solve the following two coupled equations:

$$
\begin{aligned}
1-\cos \left(\theta_{2}\right) \cosh \left(\theta_{1}\right) & =m\left[1-\cos \left(k_{1}\right) \cosh \left(k_{2}\right)\right], \\
\sin \left(\theta_{2}\right) \sinh \left(\theta_{1}\right) & =-m \sin \left(k_{1}\right) \sinh \left(k_{2}\right),
\end{aligned}
$$

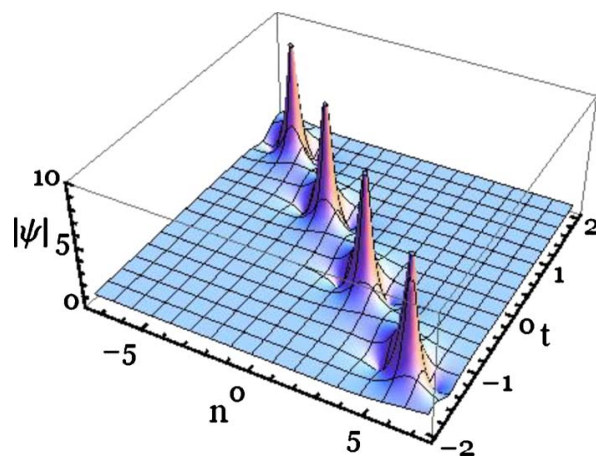

FIG. 1. (Color online) Narita solution on a background for the A-L equation presented first in [10] and given by Eq. (5) here. Although this plot shows the solution continuously along the $n$ axis, it is valid only at integer values of $n$. Here, $q=1, k_{1}=\pi / 4$, while $k_{2}=\operatorname{arccosh}(2)$ so that $\theta_{1}=1.81893$ and $\theta_{2}=-0.9546$.

$$
m=\frac{\left(1+q^{2}\right)}{q^{2}}
$$

As stated, the parameter $q$ defines the background amplitude. Two other parameters, $k_{1}$ and $k_{2}$, define the inclination of the line where the field maxima are located and their periodicity. The value of the maximum amplitude depends on this period.

We plot an example of this solution in Fig. 1 for the values of parameters $q=1, k_{1}=\pi / 4$, and $k_{2}=\operatorname{arccosh}(2)$. The conserved energy and momentum can be calculated from Eqs. (2) and (4). They are independent of $t$ and given by $Q=4.89898$ and $P=-5.16372$. Let us now consider two important limiting cases.

\section{IV. “MA SOLITON” LIMIT}

If we take the limiting case where $q$ and $k_{2}$ are arbitrary, but $k_{1} \rightarrow 0$, and hence $\theta_{2} \rightarrow 0$, then

$$
y=\frac{q^{2}}{\sinh ^{2}\left(k_{2} / 2\right)} .
$$

The relation between $\theta_{1}$ and $k_{2}$ can be found from Eq. (8), viz.,

$$
1-\cosh \left(\theta_{1}\right)=m\left[1-\cosh \left(k_{2}\right)\right],
$$

while Eq. (9) is automatically satisfied, as each side is zero. Now $w_{2} \rightarrow 0$, and

$$
w_{1}=-2 q^{2} \sinh \left(\theta_{1}\right) .
$$

We take $x_{10}=0$ and $x_{20}=0$, so that $x_{1}=w_{1} t$ and $x_{2}=k_{2} n$. In this case, solution (5) can be simplified to

$$
\psi_{n}(t)=-q \frac{\cos \left[w_{1} t+i \theta_{1}\right]+G \cosh \left(k_{2} n\right)}{\cos \left[w_{1} t\right]+G \cosh \left(k_{2} n\right)} \exp \left(2 i q^{2} t\right),
$$

where

where 


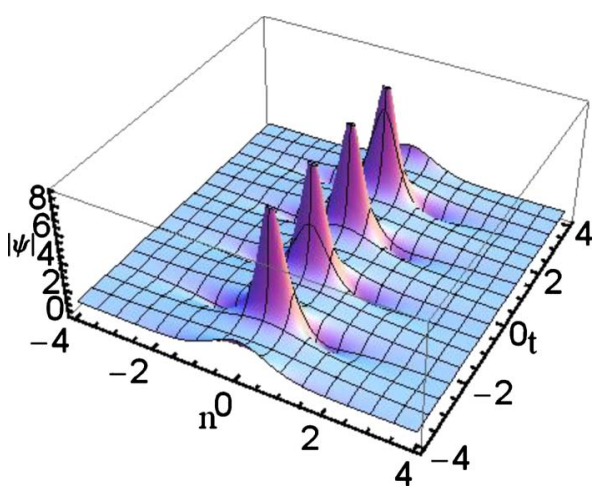

FIG. 2. (Color online) Soliton on a background defined by Eq. (10). Here, $q=1, k_{2}=1$, while $k_{1}=\theta_{2}=0$.

$$
\theta_{1}= \pm 2 \operatorname{arcsinh}\left[\sqrt{m} \sinh \left(\frac{k_{2}}{2}\right)\right]
$$

and $G$ is determined taking into account Eq. (9):

$$
G=\frac{\sinh \left(\theta_{1}\right)}{\sqrt{m} \sinh \left(k_{2}\right)}
$$

To give a more explicit form of Eq. (10), we can expand the term with the complex argument as follows:

$$
\begin{aligned}
\cos \left[w_{1} t+i \theta_{1}\right]= & {\left[1+2 m \sinh ^{2}\left(k_{2} / 2\right)\right] \cos \left[w_{1} t\right] } \\
& -2 i \sqrt{m} \sqrt{1+m \sinh ^{2}\left(\frac{k_{2}}{2}\right)} \\
& \times \sinh \left(\frac{k_{2}}{2}\right) \sin \left[w_{1} t\right] .
\end{aligned}
$$

Solution (10) is plotted in Fig. 2 for fixed values of the two independent parameters. Clearly, the background is defined by $q$, while $k_{2}$ determines the period of the structure along the $t$ axis.

As can be seen from the figure, it is a soliton on a background, with zero velocity, which propagates along the $t$ axis. Beating of the soliton with the background field leads to the periodicity in the solution. It is an analog of the $\mathrm{Ma}$ soliton in the case of the NLSE [15].

\section{Limit of the rational solution}

Solution (10) is still a one-parameter family. If we take $k_{2}$ to be small and expand $f$ and $g$ to order $k_{2}^{2}$ we obtain

$$
\psi_{n}(t)=q\left[1-\frac{4\left(1+q^{2}\right)\left(1+4 i q^{2} t\right)}{1+4 n^{2} q^{2}+16 q^{4} t^{2}\left(1+q^{2}\right)}\right] e^{2 i q^{2} t}
$$

This is the infinite period limit of the periodic solution (10), meaning that $|\psi|$ has only one peak. We note that $\psi_{0}(0)<0$. The solution is rational as it depends on the ratio of two

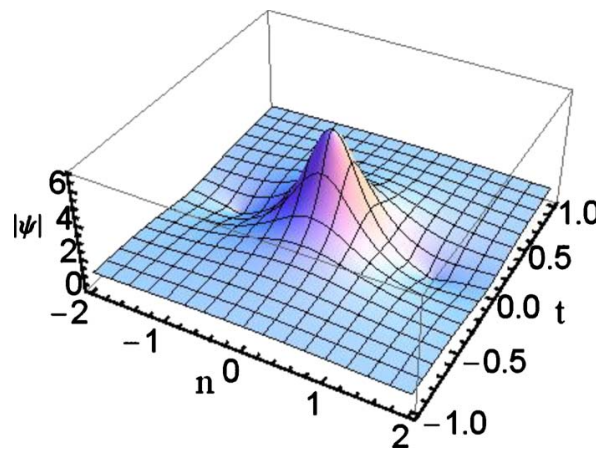

FIG. 3. (Color online) Rational solution of the first order defined by Eq. (11). Here, $q=1$, while $k_{1}=k_{2}=\theta_{2}=0$. The maximum amplitude is 7 .

polynomials. This solution is analogous to the Peregrine solution of the NLSE [16]. It is shown in Fig. 3. The resemblance to the NLSE case (see Fig. 3 of [17]) is quite remarkable.

\section{MODULATION INSTABILITY LIMIT}

In the opposite limit, when $q$ and $k_{1}$ are arbitrary but $k_{2} \rightarrow 0$ and so $\theta_{1} \rightarrow 0$, we get $w_{1} \rightarrow 0$,

$$
w_{2}=y\left\{\left[\cos \left(k_{1}\right)-1\right] \sin \left(\theta_{2}\right)\right\},
$$

where

$$
y=\frac{2\left(q^{2}+1\right)}{1-\cos \left(\theta_{2}\right)} .
$$

The relation between $\theta_{2}$ and $k_{1}$ can be found from Eq. (8), i.e.,

$$
\theta_{2}=\arccos \left\{1-m\left[1-\cos \left(k_{1}\right)\right]\right\},
$$

while Eq. (9) is automatically satisfied since each side is zero.

We take $x_{10}=0$ and $x_{20}=0$, so that $x_{1}=k_{1} n$ and $x_{2}=w_{2} t$, while

$$
w_{2}=-2 \sqrt{q^{4}-\left[\left(1+q^{2}\right) \cos \left(k_{1}\right)-1\right]^{2}} .
$$

The solution is then given by

$$
\psi_{n}(t)=-q \frac{\cos \left[k_{1} n\right]+G \cosh \left[w_{2} t+i \theta_{2}\right]}{\cos \left[k_{1} n\right]+G \cosh \left[w_{2} t\right]} \exp \left(2 i q^{2} t\right),
$$

where $G$ is determined using Eq. (9):

$$
G= \pm \sqrt{m} \frac{\sin \left(k_{1}\right)}{\sin \left(\theta_{2}\right)} \text {. }
$$

We can write solution (12) in a more explicit form by expanding the term with complex argument as follows: 


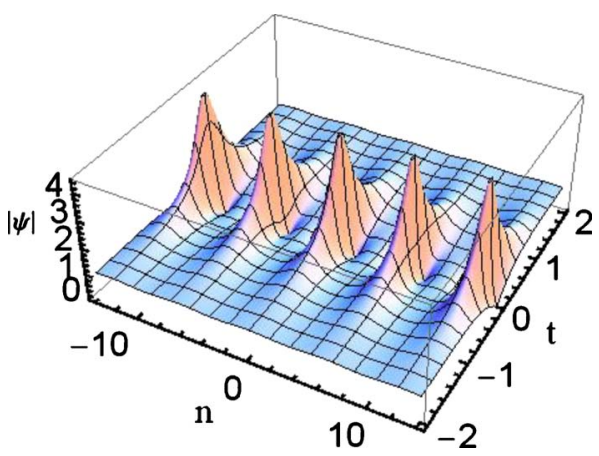

FIG. 4. (Color online) Transversely periodic solution of the A-L equation given by Eq. (12). The evolution starts with a constant background $q$, slightly modulated, then the modulation increases to reach its maximum at $t=0$ and finally the solution returns to the original background, $q$. Here, $q=1$ and $k_{1}=1$.

$$
\begin{aligned}
\cosh \left[w_{2} t+i \theta_{2}\right]= & \left\{1-m\left[1-\cosh \left(k_{1}\right)\right]\right\} \cosh \left[w_{2} t\right] \\
& +2 i \sqrt{m} \sqrt{1-m \sin ^{2}\left(\frac{k_{1}}{2}\right)} \sin \left(\frac{k_{1}}{2}\right) \sinh \left[w_{2} t\right] .
\end{aligned}
$$

This solution also has two free parameters, viz., $q$ and $k_{1}$. The value of $q$ defines the background field while $k_{1}$ defines the period of the structure in the transverse direction. An example is shown in Fig. 4 for fixed values of $q$ and $k_{1}$.

It can be seen from Fig. 4 that solution (12) starts (at $t=-\infty$ ) with the constant background field slightly perturbed by a periodic modulation. Due to the instability, the modulation increases until it reaches its maximum value (at $t=0$ ) and decreases symmetrically afterward. Thus, the solution of Eq. (12) is analogous to the solution of the NLSE commonly known as an Akhmediev breather [18-22]. The latter is the closed form solution of the NLSE whose initial stage of evolution corresponds to the growth of a modulationally unstable background [23]. It is quite instructive to compare Fig. 4 with Fig. 1 of Ref. [17].

It is worth noting here that the solution of Eq. (12) is, in fact, the complete solution of the Fermi-Pasta-Ulam (FPU) paradox for a system modeled by the A-L equation. Namely, the evolution starts with a single mode of the discrete chain, spreads into a multiplicity of spectral components at $t=0$, and then returns to the original single mode state afterward. Moreover, the solution of Eq. (12) represents FPU recurrence for a discrete system, rather than a continuous one, just like that in the original work of FPU [24], which dealt with a discrete set of oscillators. More clarifications in this regard can be found in [25], while experimental results in fiber optics are presented in [26].

\section{A. Limit giving the rational solution}

The long-period limit of solution (12) can be obtained by taking $k_{1}$ to be small. In this case we find $\theta_{2} \rightarrow \sqrt{m} k_{1}$, while $w_{2} \rightarrow-2 q \sqrt{1+q^{2}} k_{1}$. Choosing the minus sign for $G$ and expanding $f$ and $g$ to order $k_{1}^{2}$, we again get the rational solution [Eq. (11)]. Thus, both the modulation instability limit and the Ma soliton limit produce the same rational solution of the A-L equation. Similar limits can be also obtained in the case of the NLSE. A simple diagram explaining the relation between these solutions is presented in Fig. 1 of [12].

\section{B. Another reduction}

A transversely periodic solution similar to Eq. (12) has also been obtained in the recent work [11] [see Eq. (20) and Fig. 2 of that work]. However, there is a major difference between these two solutions. To see this, we can specify $k_{1}=\arccos \left[1 /\left(1+q^{2}\right)\right]$, which we label as $r$ (so $r=k_{1}$ ), thus reducing the number of parameters to just one, viz., $q$. Now, $w_{2}=-2 q^{2}$, while $\theta_{2}=\pi / 2$ and

$$
G=\sqrt{\frac{2+q^{2}}{1+q^{2}}}
$$

In this case, the solution of Eq. (12) can be significantly simplified to

$$
\psi_{n}=-q \frac{\cos (r n) \mp i \sqrt{\frac{2+q^{2}}{1+q^{2}}} \sinh \left(2 q^{2} t\right)}{\cos (r n) \pm \sqrt{\frac{2+q^{2}}{1+q^{2}}} \cosh \left(2 q^{2} t\right)} e^{2 i q^{2} t} .
$$

As expected, this solution has $q$ as its only free parameter. It defines both the background level and the transverse period along the $n$ axis. Of course, we can replace $n$ with $n-n_{0}$ to set the maximum at the origin. We can further transform this solution by dividing the numerator and denominator simultaneously by $\cosh \left(2 q^{2} t\right)$. Then after simple calculations, taking the upper signs in Eq. (13), noting $G=\sqrt{2} \cos (r / 2)$, and setting the shift to be $n_{0}=\pi / r$, we find

$$
\psi_{n}(t)=q \frac{\operatorname{sech}(s t) \cos (r n)+i \sqrt{2} \cos (r / 2) \tanh (s t)}{\sqrt{2} \cos (r / 2)-\operatorname{sech}(s t) \cos (r n)} e^{i 2 q^{2} t}
$$

where $s=2 q^{2}$. This is exactly the form of the solution derived by Chow et al. [11] by taking limits of the doubly periodic solutions which involve Jacobi elliptic functions. The maximum amplitude of this solution is

$$
\psi_{0}(0)=\frac{q \sqrt{1+q^{2}}}{\sqrt{2+q^{2}}-\sqrt{1+q^{2}}} .
$$

Thus, when $q \rightarrow 0$, the solution disappears. This solution does not produce the limit of the rational solution of the A-L equation as the two-parameter family (12). In addition, solution (14) does not describe the growth of modulation instability, as the background and the period of perturbation cannot be taken as independent. Thus, in order to keep the generality of the solution, we need to retain the form of Eq. (12), rather than Eq. (14).

\section{RATIONAL SOLUTIONS IN MORE DETAIL}

The lowest-order rational solution comes naturally from the family of Narita solutions as a limiting case with long period. Using an analogy with the NLSE, we can imagine that higher-order rational solutions may also exist. They can 

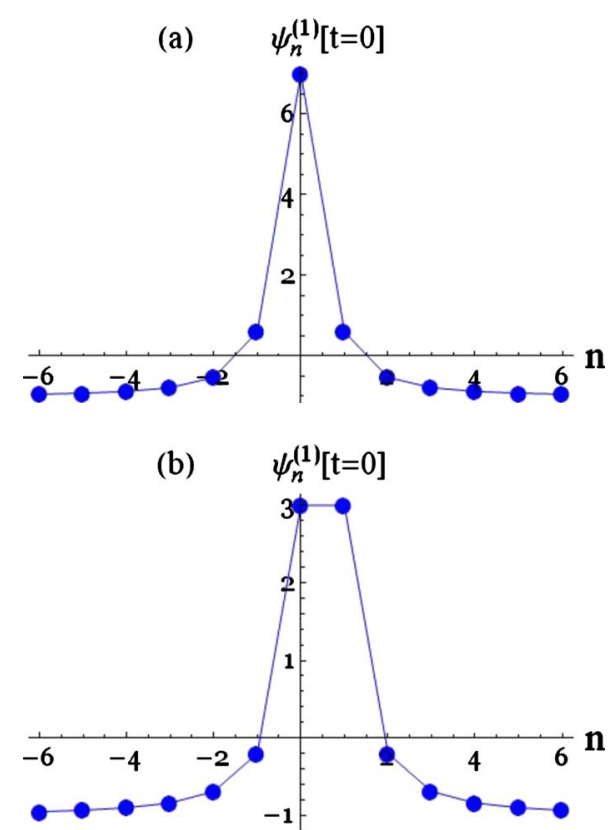

FIG. 5. (Color online) The first-order $(j=1)$ rational solution of the A-L equation given by Eq. (16) for $t=0$. (a) The offset is zero, i.e., $t_{0}=0$, and $n_{0}=0$ and $q=1$. The maximum amplitude here is 7 . (b) The offset is $t_{0}=0$, but $n_{0}=1 / 2$. The maximum amplitude is reduced to 3 compared to case (a).

be derived independently, using a bilinear Hirota-type approach. We suppose that a whole hierarchy of rational solutions with increasing order does exist. Then, we can write them all with a single expression:

$$
\psi_{n}^{(j)}(t)=q\left[4\left(1+q^{2}\right) \frac{G_{n}^{(j)}(t)+4 i q^{2} t H_{n}^{(j)}(t)}{D_{n}^{(j)}(t)}+(-1)^{j}\right] e^{2 i q^{2} t},
$$

where $j=1,2,3, \ldots$ is the order of the solution and where $G_{n}^{(j)}, H_{n}^{(j)}$, and $D_{n}^{(j)}$ are polynomials in $t$. In writing Eq. (15), we assume that $D_{n}^{(j)}$ has no real zeros. The form of the solution that we have chosen ensures that $\psi_{n}^{(j)}(0)$ is real and that its value at the origin $\psi_{0}^{(j)}(0)$ is positive.

First, we concentrate on the first-order rational solution. In order to conveniently show up the scaling factors, we define

$$
\begin{gathered}
N(n)=2 q n, \\
T(t)=4 q^{2} \sqrt{1+q^{2}} t .
\end{gathered}
$$

The first-order $(j=1)$ solution can then be written as follows:

$$
\psi_{n}^{(1)}(t)=q\left[4\left(1+q^{2}\right) \frac{G_{n}^{(1)}(t)+4 i q^{2} t H_{n}^{(1)}(t)}{D_{n}^{(1)}(t)}-1\right] e^{2 i q^{2} t},
$$

where

$$
\begin{aligned}
& G_{n}^{(1)}(t)=1, \\
& H_{n}^{(1)}(t)=1,
\end{aligned}
$$

$$
D_{n}^{(1)}(t)=1+N^{2}+T^{2} .
$$

This form agrees with the limiting case (11) found above.

Generally, we allow the translations $n \rightarrow n-n_{0}$ and $t \rightarrow t-t_{0}$, where $n_{0}$ and $t_{0}$ are arbitrary real numbers. Two forms of the solution at $t=0$ are shown in Fig. 5. The central maximum, which occurs at $t=n=0$, is $\left|\psi_{0}^{(1)}(0)\right|$, and it equals $q\left(3+4 q^{2}\right)$. The maximum is always higher than three times the background level $q$. For comparison, we note that the NLSE first-order rogue wave maximum is exactly three times the background level [17].

For $t_{0}=0$ and unit background $(q=1)$, we have

$$
\psi_{n}^{(1)}(t)=\left[\frac{8(1+4 i t)}{1+4\left(n-n_{0}\right)^{2}+32 t^{2}}-1\right] e^{2 i t} .
$$

The central maximum is then 7 as can be seen from Fig. 5(a). An offset of $\frac{1}{2}$ reduces it to $\psi_{0}^{(1)}(0)=\psi_{1}^{(1)}(0)=3$. This case is shown in Fig. 5(b). The two zeros on the line $t=0$ occur when $\left(n-n_{0}\right)^{2}=\frac{3}{4}$, so it can happen that a zero does occur for integer $n$. For example, node $n=0$ is a zero if $n_{0}=\frac{\sqrt{3}}{2}$.

\section{A. Generalization: Rational solution of the discrete Hirota equation}

Following Narita [3], we can also introduce the discrete Hirota equation

$$
\left.i \frac{\partial \psi_{n}}{\partial t}+\left[(a-i b) \psi_{n-1}+(a+i b) \psi_{n+1}\right)\right]\left(1+\left|\psi_{n}\right|^{2}\right)-2 \psi_{n}=0,
$$

where $a$ and $b$ are arbitrary real numbers. Similar to the continuous Hirota equation [27], this generalization of Eq. (1) has two limiting cases. It is reduced to the A-L equation (1) when $a=1$ and $b=0$, while it reduces to the discrete $\mathrm{mKdV}$ equation

$$
\frac{\partial \psi_{n}}{\partial t}+\left(\psi_{n+1}-\psi_{n-1}\right)\left(1+\left|\psi_{n}\right|^{2}\right)=0
$$

when $a=0$ and $b=1$ [3]. In fact, the solutions [Eqs. (5) and (20)] presented above are retained for arbitrary values of $a$ and $b$.

Thus, for arbitrary real values of $a, b$, and $q$, we can write the rational solution to Eq. (21) as follows:

$$
\psi_{n}^{(j)}=q\left[4\left(1+q^{2}\right) \frac{G_{n}^{(j)}(t)+\frac{i T}{\sqrt{1+q^{2}}} H_{n}^{(j)}(t)}{D_{n}^{(j)}(t)}+(-1)^{j}\right] e^{i \phi},
$$

where $j=1$,

$$
\phi=2 t\left[\left(1+q^{2}\right) \sqrt{a^{2}+b^{2}}-a\right]+n \arctan (b / a),
$$

and $N(n)=2 q n$ is retained as before, but we have generalized the value of $T(t)$ to

$$
T(t)=4 q^{2} \sqrt{a^{2}+b^{2}} \sqrt{1+q^{2}} t .
$$

For $j=1$, the forms of $G_{n}^{(j)}(t), H_{n}^{(j)}(t)$, and $D_{n}^{(j)}(t)$ are the same as in Eqs. (17) and (19). If $a=0$, we use $\arctan (b / a)=\pi / 2$. 
Clearly, for the A-L equation $(a=1$ and $b=0)$, Eq. (23) reduces to Eq. (15).

\section{B. Rogue wave conserved quantities}

For the rogue wave $\psi_{n}$ of order $j$ and background amplitude $q$, we use the definitions given by Eqs. (2) and (4). For $q=1$, we find

$$
Q_{n}^{(1)}=\frac{16(n+1)}{1+4(n+1)^{2}+32 t^{2}}-\frac{16 n}{1+4 n^{2}+32 t^{2}} .
$$

This expression is symmetric on the line $n=-1 / 2$ for all $t$. Changing the variable $n+1 \rightarrow m$ in the first part of Eq. (25), we can see that the two summations are exactly equal. Thus, they subtract to zero, showing that energy is conserved for all $t$, viz., $Q^{(1)}=0$. The momentum is

$$
P^{(1)}=64 t \sum_{n=-\infty}^{\infty}\left(\frac{1}{1+4 n^{2}+32 t^{2}}-\frac{1}{1+4(n+1)^{2}+32 t^{2}}\right),
$$

which is clearly zero for all $t$, showing that momentum is also conserved.

\section{SECOND-ORDER ROGUE WAVE}

As we have seen from the previous analysis, the Narita solutions to some extent play the role of first-order solitons of the NLSE [28]. Thus, detailed knowledge of its properties is essential for constructing higher-order solutions. Following the example of the first-order solution, we can present the second-order one $(j=2)$ as a one-parameter family with arbitrary background level $q$ :

$$
\psi_{n}^{(2)}(t)=q\left[4\left(1+q^{2}\right) \frac{G_{n}^{(2)}(t)+i 4 q^{2} t H_{n}^{(2)}(t)}{D_{n}^{(2)}(t)}+1\right] e^{2 i q^{2} t}
$$

where

$$
\begin{aligned}
G_{n}^{(2)}(t)= & 3\left[3-6\left(N^{2}-2 q^{2}\right)\right. \\
& \left.+N^{2}\left(4 q^{2}-N^{2}\right)-6\left(3+2 q^{2}+N^{2}\right) T^{2}-5 T^{4}\right],
\end{aligned}
$$

$$
\begin{aligned}
H_{n}^{(2)}(t)= & 3\left[15+6\left(N^{2}+4 q^{2}\right)\right. \\
& \left.+N^{2}\left(16 q^{2}-N^{2}\right)-2\left(1+N^{2}\right) T^{2}-T^{4}\right],
\end{aligned}
$$

while

$$
\begin{aligned}
D_{n}^{(2)}(t)= & 9+\left(27+24 q^{2}+16 q^{4}\right) N^{2}+N^{4}\left(3-8 q^{2}\right) \\
& +N^{6}+3\left(33+72 q^{2}-6 N^{2}+48 q^{4}-16 q^{2} N^{2}+N^{4}\right) T^{2} \\
& +3\left(9+8 q^{2}+N^{2}\right) T^{4}+T^{6} .
\end{aligned}
$$

Here, we have used $N(n)=2 q n$ and $T(t)=4 q^{2} \sqrt{1+q^{2}} t$, so we have the same scaling as in previous sections. An example of solution (27) with $q=1 / 4$ is plotted in Fig. 6. In contrast to the previous examples, we have chosen a relatively small value of $q$, viz., $q=0.25$, as in this case the solution is less

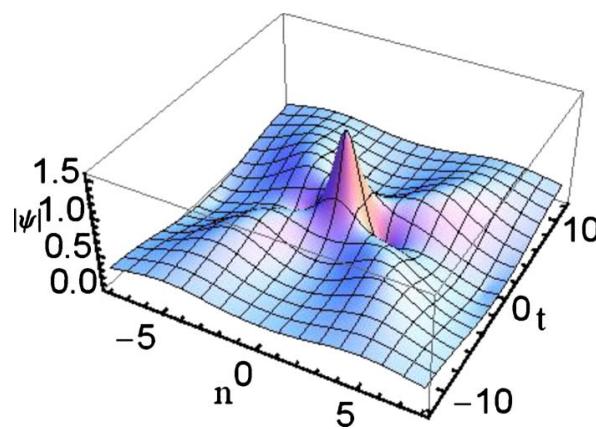

FIG. 6. (Color online) The second-order $(j=2)$ rational A-L solution [Eq. (27)], for $q=1 / 4$ with zero offset $\left(n_{0}=0\right)$.

peaked and covers more sites than in the $q=1$ case. A quick glance at Fig. 6 shows that it has a striking resemblance to the second-order rational solution of the NLSE (see Fig. 4 of [17]).

As the background level is $q$, then for zero offset $\left(n_{0}=0\right)$, the central maximum is

$$
\psi_{0}^{(2)}(0)=q\left(5+20 q^{2}+16 q^{4}\right)
$$

This means that the maximum amplitude is always more than five times the background level. For comparison, the NLSE second-order rogue wave maximum is exactly five times the background level $[17,29]$. As $q$ increases, the distribution becomes more sharply peaked around the origin, and the maximum amplitude increases rapidly (Fig. 7). In fact, the height of the maximum amplitude above the background level is $\psi_{0}^{(2)}(0)-q=4 q\left(1+q^{2}\right)\left(1+4 q^{2}\right)$.

The solution with the unit background is found by taking $q=1$. Then $N=n$ and $T=\sqrt{2} t$. The maximum amplitude at the central point in this case is 41. As a function of $n, \psi_{n}^{(2)}(t=0, n)$ has four zeros. An offset of $\frac{1}{2}$ produces a considerable change-the maximum is reduced to $\psi_{0}^{(2)}(0)=\psi_{1}^{(2)}(0)=5 q$, as shown in Fig. 8. Despite the nonzero offset, $Q_{n}^{(2)}(t)$ is symmetric in the line $n=1 / 2$ for all $t$. Thus, it is easy to see that energy is conserved, viz., $Q^{(2)}=0$. Similarly, momentum is also conserved $P^{(2)}=0$.

The same polynomials given by Eqs. (28)-(30) provide the solution to the Hirota equation [Eq. (21)]. In this case $j=2$ in Eq. (23), with $T(t)$ given by Eq. (24).

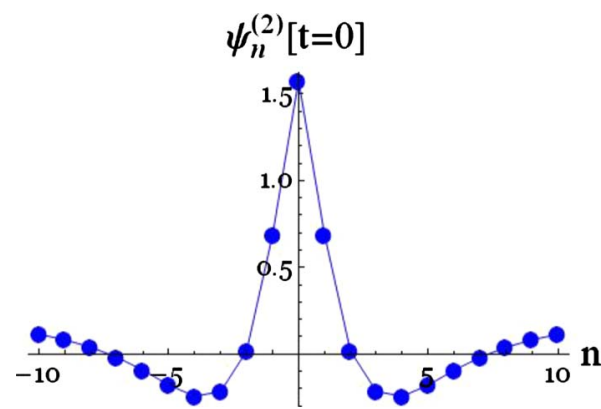

FIG. 7. (Color online) Plot of the second-order $(j=2)$ rational solution of the A-L equation given by Eq. (27) at $t_{0}=0$. The background level $q=1 / 4$ and the offset $n_{0}=0$. 


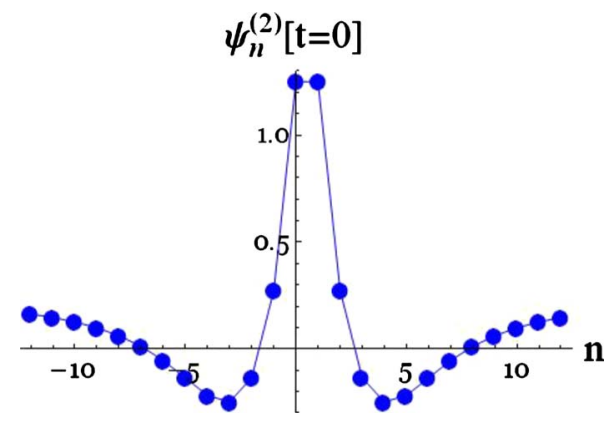

FIG. 8. (Color online) Plot of the second-order $(j=2)$ rational solution of the A-L equation given by Eq. (27) at $t_{0}=0$. The background level $q=1 / 4$ and the offset $n_{0}=1 / 2$. The central maximum in this case is reduced to $\psi_{0}^{(2)}(0)=\psi_{1}^{(2)}(0)=5 / 4$.

\section{CONCLUSION}

We have found the second-order rational solution for the discrete A-L equation. This solution can be used as an approximation for rogue waves of light in an array of strongly coupled optical waveguides [14]. Depending on the param- eters of the system, the light can be concentrated into very strong peaks.

Our main result here is that higher-order rational solutions exist for the discrete A-L equation and exhibit an analogy with rogue waves of the continuous NLSE [30]. However, the coefficients and scaling in the expressions for the rational solutions for the discrete case are quite different from those found for the NLSE $[17,31]$. The most remarkable feature of a discrete rogue wave is that the height of the central maximum relative to the background is much greater than in the corresponding NLSE case. More generally, we have presented rational solutions for the discrete Hirota equation, which includes, as particular cases, both the discrete A-L equation and the discrete $\mathrm{mKdV}$ equation.

\section{ACKNOWLEDGMENTS}

The two authors A.A. and N.A. acknowledge the support of the A.R.C. (Discovery Project No. DP0985394). J.M.S.C. acknowledges support from the Spanish Ministerio de Ciencia e Innovación under Contracts No. FIS2006-03376 and No. FIS2009-09895.
[1] M. J. Ablowitz and J. F. Ladik, Stud. Appl. Math. 55, 213 (1976).

[2] M. J. Ablowitz and J. F. Ladik, J. Math. Phys. 17, 1011 (1976).

[3] K. Narita, J. Phys. Soc. Jpn. 59, 3528 (1990).

[4] V. E. Vekslerchik and V. Konotop, Inverse Probl. 8, 889 (1992).

[5] K. Maruno and Y. Ohta, J. Phys. Soc. Jpn. 75, 054002 (2006).

[6] V. E. Vekslerchik, J. Nonlinear Math. Phys. 9, 157 (2002).

[7] Qin Zhenyun, J. Math. Phys. 49, 063505 (2008).

[8] X. Liu and Y. Zeng, J. Phys. A 40, 8765 (2007).

[9] P. D. Miller et al., Commun. Pure Appl. Math. 48, 1369 (1995).

[10] K. Narita, J. Phys. Soc. Jpn. 60, 1497 (1991).

[11] K. Chow, R. Conte, and N. Xu, Phys. Lett. A 349, 422 (2006).

[12] N. Akhmediev, J. M. Soto-Crespo, and A. Ankiewicz, Phys. Lett. A 373, 2137 (2009).

[13] S. Flach and A. Gorbach, Phys. Rep. 467, 1 (2008).

[14] Yu. V. Bludov, V. V. Konotop, and N. Akhmediev, Opt. Lett. 34, 3015 (2009).

[15] Ya.-C. Ma, Stud. Appl. Math. 60, 43 (1979).

[16] D. H. Peregrine, J. Aust. Math. Soc. Ser. B, Appl. Math. 25, 16 (1983).

[17] N. Akhmediev, A. Ankiewicz, and M. Taki, Phys. Lett. A 373, 675 (2009).

[18] K. B. Dysthe and K. Trulsen, Phys. Scr. T82, 48 (1999).

[19] D. Clamond, M. Francius, J. Grue, and C. Kharif, Eur. J.
Mech. B/Fluids 25, 536 (2006).

[20] I. Ten and H. Tomita, Kyushu University Reports of RIAM Symposium No. 17SP1-2, 2006 (unpublished).

[21] V. V. Voronovich, V. I. Shrira, and G. Thomas, J. Fluid Mech. 604, 263 (2008).

[22] V. I. Shrira and V. V. Geogjaev, J. Eng. Math. 67, 11 (2010).

[23] N. Akhmediev and V. I. Korneev, Teor. Mat. Fiz. 69, 189 (1986) [Theor. Math. Phys. 69, 1089 (1986)].

[24] E. Fermi, J. Pasta, and S. Ulam, Los Alamos National Laboratory Report No. LA-1940, 1955 (unpublished); reprinted in E. Fermi Collected Papers, edited by E. Segre (University of Chicago Press, Chicago, IL, 1965), Vol. II, p. 978.

[25] N. Akhmediev, Nature (London) 413, 267 (2001).

[26] G. Van Simaeys, Ph. Emplit, and M. Haelterman, Phys. Rev. Lett. 87, 033902 (2001).

[27] A. Ankiewicz, J. M. Soto-Crespo, and N. Akhmediev, Phys. Rev. E 81, 046602 (2010).

[28] N. Akhmediev, V. M. Eleonskii, and N. E. Kulagin, Teor. Mat. Fiz. 72, 183 (1987) [Theor. Math. Phys. 72, 809 (1987)].

[29] A. Ankiewicz, http://demonstrations.wolfram.com/ RogueOceanWaves/

[30] N. Akhmediev, J. M. Soto-Crespo, and A. Ankiewicz, Phys. Rev. A 80, 043818 (2009); see also M. Marklund, and L. Stenflo, Physics 2, 86 (2009).

[31] N. Akhmediev, A. Ankiewicz, and J. M. Soto-Crespo, Phys. Rev. E 80, 026601 (2009). 